Invest. Pens. Crit. (ISSN 1812-3864; eISSN 2644-4119)

DOI: https://doi.org/10.37387/ipc.v9i1.209

Vol. 9, No. 1, Enero - Abril 2021

pp. 31 - 45

\title{
E1 TERCE en Panamá: una mirada a los logros de aprendizaje y factores asociados
}

\author{
Daysi Jackson ${ }^{1}$, Rosa Arrocha ${ }^{2}$, Jaime Estrella ${ }^{3}$ \\ ${ }^{1}$ Docente, Colegio San Carlos, Ministerio de Educación. Panamá. \\ ${ }^{2}$ Docente, Departamento de Estadística, Facultad de Economía, Universidad de Panamá. Panamá. \\ ${ }^{3}$ Investigador independiente, Especialista en gestión de investigación. Panamá.
}

*Autor para correspondencia. E-mail: daysinoemi_jacksontapia@hotmail.com

Recibido: 07 de septiembre de 2020

Aceptado: 20 de octubre de 2020

\section{Resumen}

Este artículo muestra los resultados de Panamá de un estudio mixto, que en su etapa inicial utilizó los datos generados por el Tercer Estudio Regional Comparativo Explicativo (TERCE) llevado a cabo por el Laboratorio Latinoamericano de Evaluación de la Calidad de la Educación (LLECE) de la UNESCO. Nuestra investigación muestra los resultados promedios de las pruebas, los niveles de desempeño y factores asociados. Se cuantificó el efecto de las escuelas en la variabilidad de los logros de aprendizaje, a través de un modelo regresión multinivel. Se estudió la asociación entre el logro de aprendizaje y las características de estudiantes, docentes y directores, mediante el Coeficiente de Correlación de Pearson para las variables cuantitativas, mientras que para las variables cualitativas se empleó el Coeficiente de Gamma. En relación a los factores asociados, a nivel de alumno, los de incidencia negativa fueron repitencia y si el alumno trabaja. En contraste, los de incidencia positiva fueron tener libros y cuadernos, la motivación de los maestros hacia sus alumnos de continuar estudiando y la explicación que el maestro da a sus alumnos en clase. El estudio permitió hacer algunas recomendaciones para mejorar el aprendizaje efectivo en Panamá.

Palabras clave: logros de aprendizaje, niveles de desempeño, educación, factores asociados, TERCE Panamá.

\begin{abstract}
This article shows the results of Panama from a mixed study, which in its first stage used the data generated by the Third Regional Explanatory Comparative Study (TERCE after its acronym in Spanish) conducted by the Laboratorio Latinoamericano de Evaluación de la Calidad de la Educación (LLECE in Spanish) from UNESCO. Our research shows the average test results, performance levels and associated factors. The effect of schools on the variability of learning achievement through a multilevel regression model was quantified. The association between learning achievement and the characteristics of students, teachers and principals was studied using Pearson Correlation Coefficient for
\end{abstract}


quantitative variables; meanwhile, for qualitative variables the Gamma Coefficient was used. In relation to the associated factors, at the student level, the negative incidence was repetition and if the student works. Factors of positive incidence were identified as having books and notebooks, the motivation of the teachers towards their students to continue studying, and the explanation that teachers give to their students during class. Our study allows recommendations to improve effective learning in Panama.

Keywords: learning achievements, performance levels, education, associated factors, TERCE Panamá.

\section{Introducción}

Diversos estudios derivados de evaluaciones nacionales, regionales e internacionales se han realizado en numerosos países; uno de ellos fue el denominado "Las escuelas que hacen la diferencia: evidencia de la prueba TERCE 2013 en la construcción de comunidades enfocadas en el aprendizaje”, desarrollado por la Agencia de la Calidad de la Educación (2015). Su finalidad fue comprender qué hacen los diferentes actores de la comunidad educativa (estudiantes, padres de familia, maestros y directivos) para promover acciones hacia la mejora. El mismo estudio caracterizó aquellos actores que lograban interacciones favorables hacia el aprendizaje. La metodología utilizada fue análisis de regresión jerárquica en el nivel estudiante anidado en el nivel escuela.

Un estudio similar fue "¿La escuela o la cuna? Evidencias sobre su aportación al rendimiento de los estudiantes de América Latina: Estudio multinivel sobre la estimación de los efectos escolares". Este estudio utilizó la base de datos del Segundo Estudio Regional Explicativo y Comparativo (SERCE) de la UNESCO/OREALC (2009), para estimar los efectos escolares en los rendimientos que alcanzaron en lectura y matemática de los estudiantes latinoamericanos de $3^{\circ}$ y $6^{\circ}$ grado de primaria, empleando modelos multinivel. El propósito del estudio fue determinar si son la escuela o el hogar los que influyen en los logros de aprendizaje y si esto es explicado por la varianza del nivel cultural y socioeconómico de las familias en dichos rendimientos (Torrecilla \& Carrasco, 2011).

Murillo y Hernández (2011) centraron su investigación en los efectos escolares sobre variables socioafectivas tales como: autoconcepto, disciplina, convivencia social y satisfacción con la escuela. Entre los resultados obtenidos es posible mencionar que estudiantes de grupos indígenas tenían menor autoconcepto y satisfacción por la escuela, no así estudiantes de un nivel socioeconómico alto que mostraron un mejor autoconcepto, mejor comportamiento, se adaptan mejor y se sienten satisfechos con la escuela. Uno de los resultados destacables del mismo estudio fue que la escuela tiene una importancia muy pequeña en el desarrollo de productos socio-afectivos (2011).

El estudio "Pistas hechas en Latinoamérica ¿Qué hicieron los países, escuelas y estudiantes con mejor desempeño en el Tercer Estudio Regional Comparativo y Explicativo (TERCE)?”, de Ganimiam (2016), responde a la pregunta ¿qué políticas educativas se relacionan con mejores resultados en una prueba latinoamericana

1 TERCE: Tercer Estudio Regional Comparativo y Explicativo, realizado por el Laboratorio Latinoamericano de la Evaluación de la Calidad de la Educación (LLECE), coordinado por la Oficina Regional de Educación de la UNESCO para América Latina y el Caribe (OREALC/UNESCO, Santiago de Chile). 
de desempeño estudiantil? Una de las conclusiones de ese estudio fue que en América Latina hay una diversidad de políticas educativas que coexisten; y, esto deja ver que existen diversos "caminos" para la mejora de la calidad educativa, por ejemplo, en países como Costa Rica y Chile, que presentan políticas en educación muy distintas, y que dan testimonio sobre esta diversidad.

La investigación cuantitativa descrita más adelante corresponde a la primera etapa de una investigación financiada por la Secretaría Nacional de Ciencia, Tecnología e Innovación (SENACY'T, Panamá), bajo el nombre "Escuelas que hacen la diferencia en la República de Panamá: el TERCE y un estudio en escuelas oficiales en contextos desfavorables que lograron resultados por encima de la media en lectura, matemática y ciencias", que en su ejecución total en campo correspondió a un estudio de corte mixto.

El objetivo de este estudio fue analizar los niveles de logro de aprendizaje en la evaluación TERCE en Panamá, así como también se identificar factores que se asocian positivamente a mayores logros de aprendizaje a nivel de estudiante, docente, hogar y escuela. Para ello, se listaron las características socioeconómicas de aquellas escuelas que se clasificaron en un nivel socioeconómico desfavorable y se identificaron las escuelas que obtuvieron un promedio arriba de la media nacional, pero teniendo un nivel socioeconómico desfavorable.

\section{El estudio TERCE y la participación de Panamá}

El TERCE (que se implementó en 2013, con la participación de 15 países de la región latinoamericana, más el Estado de Nuevo León, México), entregó información sobre la eficacia escolar y dio cuenta de las brechas en los niveles de logros de aprendizajes entre estudiantes de diferentes contextos socioculturales, con el fin de que los países puedan tener un panorama del sistema educativo que les permita reflexionar sobre lo que se hace en las escuelas y crear políticas públicas que se traduzcan en el mejoramiento de la calidad educativa.

Su evaluación consistió en medir los logros de aprendizaje en lenguaje (lectura y escritura), matemática en tercero y sexto grados, y ciencias en sexto grado. Además, se identificó factores asociados a los logros de aprendizaje. Para ello, el TERCE utilizó dos tipos de instrumentos de recolección de datos: pruebas de evaluación de aprendizaje y cuestionarios de contexto.

Panamá participó del TERCE con una muestra representativa de 184 escuelas (137 del sector oficial y 47 del sector particular). Este es el contexto en el que se basa este estudio y los análisis estadísticos que se muestran a continuación.

\section{Materiales y métodos}

En el contexto de la participación de las escuelas de Panamá en el TERCE (arriba descrito), se compilaron los promedios de los puntajes a nivel nacional para cada una de las áreas curriculares evaluadas, y luego se calculó el promedio por sector oficial y particular. Los resultados oficiales se desagregaron por área geográfica urbana y rural. En el sector particular no se hizo esta desagregación porque sólo había una escuela del sector rural. También se calcularon los promedios por sexo para cada sector. 
Se aplicaron pruebas de hipótesis para la media, con el fin de obtener la significancia estadística de la media del país con respecto al puntaje promedio del TERCE y para comparar los promedios oficiales y particulares con el promedio TERCE. Además, se comparó las medias de las áreas geográficas en el sector oficial. Adicionalmente, se hicieron las distribuciones de frecuencias de los niveles de desempeño para cada prueba y grado, según sector oficial y particular. En los factores asociados se desarrolló el trabajo analizando el índice de nivel socioeconómico, calculando las estadísticas descriptivas estratificadas por sector (oficial / particular).

Con la finalidad de relacionar los resultados y el nivel socioeconómico, se calculó el promedio de logros académicos para los quintiles del índice de nivel socioeconómico y así mostrar el efecto de dicho nivel en logros académicos para los sectores oficiales y particulares. Por otro lado, para cuantificar el efecto de las escuelas en la variabilidad de los logros de aprendizaje, se aplicó la técnica de modelos multinivel y se estimaron modelos nulos con efectos aleatorios.

Para estudiar la asociación entre los logros de aprendizaje y las características de los estudiantes, docentes y directores se calcularon coeficientes de correlación con sus respectivas significancias. Para variables cuantitativas se usó el Coeficiente de Correlación de Pearson (Warren, 1971; Sancho et al., 2014). Para las variables cualitativas se usó el Coeficiente de Eta o Gamma (incluido en StataCorp, 2013), para lo cual el puntaje de los estudiantes fue categorizado en cuatros grupos considerando los cuartiles en las diferentes pruebas de cada nivel.

Para las variables donde hubo variabilidad en las diferentes categorías, se calcularon los promedios de los puntajes de las diferentes pruebas, para cada una de las categorías, a fin de mostrar asociación positiva o negativa y visibilizar el tipo de relación.

A través de un modelo multinivel de efectos aleatorios se cuantificó el aporte de las escuelas al aprendizaje de los estudiantes. Se estimó el indicador de variación del resultado de la prueba que corresponde a la variación entre escuelas, conocido como el índice de correlación intra-clase (ICC), que indica el peso relativo del grupo de escuelas para explicar el valor que toma la variable dependiente (puntaje de las pruebas).

Esto implica que, a mayor ICC, más grande es la similitud de los resultados al interior de la escuela, en relación con los resultados de otras escuelas. En otras palabras, el índice indica cuán importante es lo que hace la escuela para influenciar el logro de aprendizaje, por sobre la influencia de las características individuales y familiares.

Este procedimiento se realizó mediante la estimación de un modelo nulo o sin variables independientes, que divide la varianza total no condicional en dos partes que suman entre sí: la varianza entre individuos y la varianza entre grupos o escuelas, a partir de las cuales se calculó el ICC. El porcesamiento de datos fue realizado con el software STATA 13 (StataCorp, 2013). 


\section{Resultados}

\section{Logros de aprendizaje}

Los resultados del TERCE en Panamá se muestran a continuación de dos maneras: (i) por puntajes promedios por escuela, que centran en una media de 700 puntos y una desviación estándar de 100 puntos; y, (ii) por niveles de desempeño ${ }^{2}$.

\section{Puntaje promedio en las pruebas de lectura, matemática y ciencias}

El promedio de los puntajes en la prueba de lectura para tercer grado de Panamá fue de 671 y es significativamente inferior al promedio TERCE, que fue de 700 puntos para toda la región latinoamericana.

Al calcular el promedio por sector, el promedio de escuelas oficiales de Panamá fue de 659, que es significativamente inferior al promedio TERCE regional, mientras que el promedio de escuelas particulares de Panamá fue de 755, que es significativamente superior al promedio regional TERCE. En el país, para el sector oficial, los promedios de las áreas geográficas fueron: en las escuelas del área urbana fue de 687, que es significativamente superior a las escuelas del área rural, con 653 puntos.

En el caso de la prueba de lectura para sexto grado en Panamá fue de 670 y es significativamente inferior al promedio TERCE de la región. Al calcular el promedio por sector en Panamá, el promedio de escuelas oficiales fue de 660 y es significativamente inferior al promedio TERCE regional, mientras que el promedio de las escuelas particulares fue de 755, que es significativamente superior al promedio regional TERCE. Para el sector oficial, en Panamá, en cuanto a áreas geográficas, el promedio de las escuelas del área urbana fue de 684 y es significativamente superior a las escuelas del área rural con 651 puntos.

En la prueba de matemática en tercer grado, el puntaje promedio fue de 665 y en sexto grado de 664; es decir, los promedios de los puntajes nacionales fueron significativamente menores que el puntaje regional TERCE. Al estratificar por sector oficial-particular en los dos grados, en el sector particular las escuelas obtuvieron promedios significativamente mayores al promedio TERCE, mientras que en el sector oficial los promedios de las escuelas fueron significativamente menores al promedio TERCE. En el sector oficial en tercer grado, el área urbana tiene 31.73 puntos más que el área rural. Por su parte, en la prueba de sexto, el área urbana obtuvo 12.7 puntos más que el área rural.

En ciencias también se observó que el promedio particular fue significativamente mayor al promedio TERCE y el promedio oficial significativamente menor al promedio TERCE. El promedio del estrato oficial urbano fue significativamente mayor al oficial rural, esto es 30.1 puntos más (Tabla 1).

\footnotetext{
${ }^{2}$ Niveles de desempeño, definidos para el TERCE, por el LLECE (UNESCO), son descriptores de las habilidades cognitivas que tienen los estudiantes en lectura, matemática y ciencias. El nivel más bajo es el I, le siguen el II, III y el más alto es el nivel IV. En nuestro análisis sólo se mencionan algunos de ellos.
} 
Tabla 1.

Puntuaciones promedio en la prueba de lectura, matemática y ciencias naturales de tercero y sexto grados, según sector, área geográfica y sexo.

\begin{tabular}{|c|c|c|c|c|c|c|c|c|}
\hline \multirow{3}{*}{$\begin{array}{l}\text { Valores } \\
\text { promedio } \\
\text { Total país }\end{array}$} & \multicolumn{3}{|l|}{ Lectura } & \multicolumn{3}{|l|}{ Matemática } & \multirow{2}{*}{\multicolumn{2}{|c|}{\begin{tabular}{|l|} 
Ciencias \\
Sexto
\end{tabular}}} \\
\hline & Tercero & \multicolumn{2}{|c|}{ Sexto } & Tercero & \multicolumn{2}{|l|}{ Sexto } & & \\
\hline & $670.3 \downarrow^{*}$ & 671.4 & $\downarrow^{*}$ & $664.09 \downarrow^{*}$ & 643.9 & $\downarrow^{*}$ & 674.7 & $\downarrow *$ \\
\hline Oficial & $659.9 \downarrow^{*}$ & 658.8 & $\downarrow^{*}$ & $657.11 \downarrow^{*}$ & 638.8 & $\downarrow^{*}$ & 668.1 & $\downarrow^{*}$ \\
\hline Urbana & $686.9 \uparrow * *$ & 683.8 & $\uparrow * *$ & 681.55 个*** & 648.2 & $\uparrow * * *$ & 691 & $\uparrow * * *$ \\
\hline Niño & 679.2 & 669.4 & & 679.63 & 645.6 & & 684.7 & \\
\hline Niña & 695.7 & 696.7 & & 683.82 & 650.6 & & 696.2 & \\
\hline Rural & 652.8 & 651 & & 649.82 & 635.5 & & 660.9 & \\
\hline Niño & 679.2 & 644.9 & & 647.16 & 634.4 & & 658.9 & \\
\hline Niña & 659.3 & 656.8 & & 652.61 & 636.6 & & 662.8 & \\
\hline Particular & 755.2 个*** & 754.9 & $\uparrow * * *$ & $739.62 \quad \uparrow * *$ & 721 & $\uparrow * *$ & 762.5 & $\uparrow * *$ \\
\hline Niño & 748.2 & 746.4 & & 739.45 & 724.4 & & 758.8 & \\
\hline Niña & 761.8 & 762.6 & & 739.77 & 717.9 & & 765.7 & \\
\hline
\end{tabular}

\section{Códigos:}

$\downarrow^{*} \quad$ significativamente menor que la media del TERCE.

$\uparrow * * \quad$ significativamente mayor que la media del TERCE.

$\uparrow * * *$ diferencias significativas mayores entre los promedios del área urbana y rural (particular/oficial).

Por otro lado, en la prueba de lectura de tercer grado (Figura 1), los estudiantes del sector oficial se distribuyen en un 48\% en el nivel más bajo de desempeño; esto es el nivel I, que significa, por ejemplo, que el estudiante sólo extrae información explícita en un lugar destacado del texto; en el nivel II, el 21 $\%$ de los estudiantes comprenden el sentido global del texto a partir de información explícita, reconocida y reiterada en el texto; y, en los niveles III y IV llega a 16\% y 3\%, respectivamente.
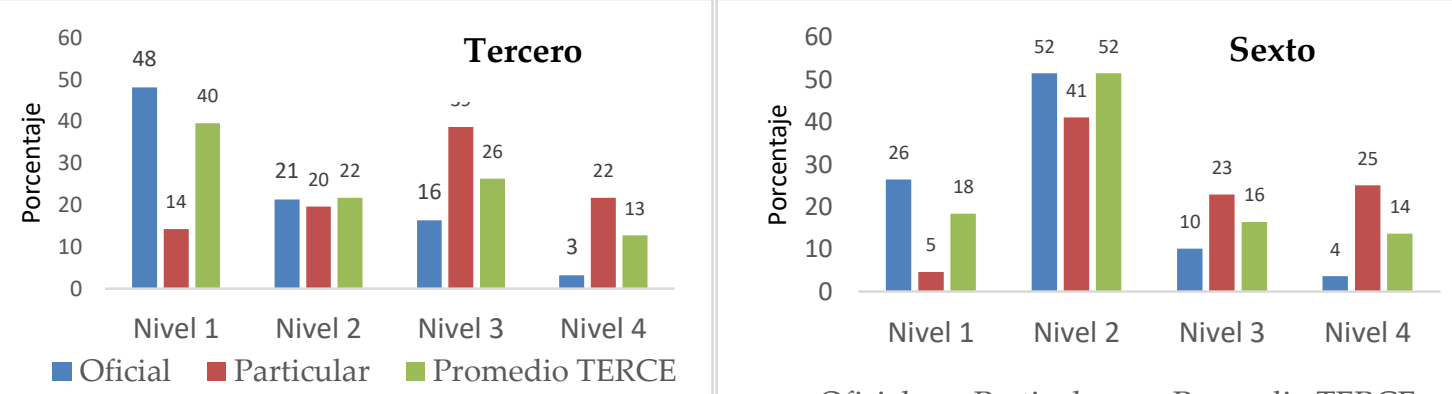

Oficial Particular Promedio TERCE

Figura 1. Distribución de los estudiantes por nivel de desempeño en la prueba de Lectura según grado en Panamá. 
Esto significa, por ejemplo, que los estudiantes distinguen el tema central y otras ideas a partir de información explícita. De igual manera ocurre en el sector particular: en el nivel I se observa 14\%, en el nivel II un 20\%, en el nivel III hay un 39\% (que es un valor superior al porcentaje promedio de todos los países participantes, de 26\%); y, en el nivel IV se observa un $22 \%$, que es superior al promedio de todos los países (que alcanzó un 13\%).

En la prueba de lectura de sexto grado (Figura 1), los estudiantes del sector oficial de Panamá se distribuyen en un 26\% en el nivel I (el más bajo). Esto significa, por ejemplo, que el alumno localiza información explícita parafraseada en el texto; en el nivel II, un 52\% infiere el significado de palabras familiares a partir de claves que da el texto. En el nivel III, un 10\% de alumnos reconoce la función de distintos elementos y recursos de un texto, o parafresea o interpreta figuras literarias; mientras tanto en el nivel IV un 4\%, por ejemplo, infiere el significado de palabras utilizadas con significados diversos.

Por su parte, en el sector particular en el nivel I se observa 5\%, en el nivel II un 41\%, en el nivel III hay un 23\% (que es un valor superior al porcentaje promedio de todos los países participantes, de16\%); finalmente, en el nivel IV se observa un 25\% (valor superior al promedio de todos los países, de 14\%).

En la prueba de matemática de tercer grado (Figura 2), los estudiantes del sector oficial se distribuyen en un 63\% en el nivel I, lo que significa que sólo identifican figuras geométricas básicas, por ejemplo. En el nivel II, un 22\%, por ejemplo, lee y escribe números naturales. En el nivel III, un 9\% resuelve problemas con números naturales, mientras que en el nivel IV un 1\% resuelve problemas geométricos.

De igual manera, en el sector particular, en el nivel I se observa un 25\%; el nivel II alcanza $27 \%$ y en el nivel III hay un 33\% (que es un valor superior al porcentaje promedio de todos los países participantes, de 23\%). En el nivel IV se observa un $8 \%$ (valor ligeramente superior al promedio de todos los países, que fue de $7 \%$ ).

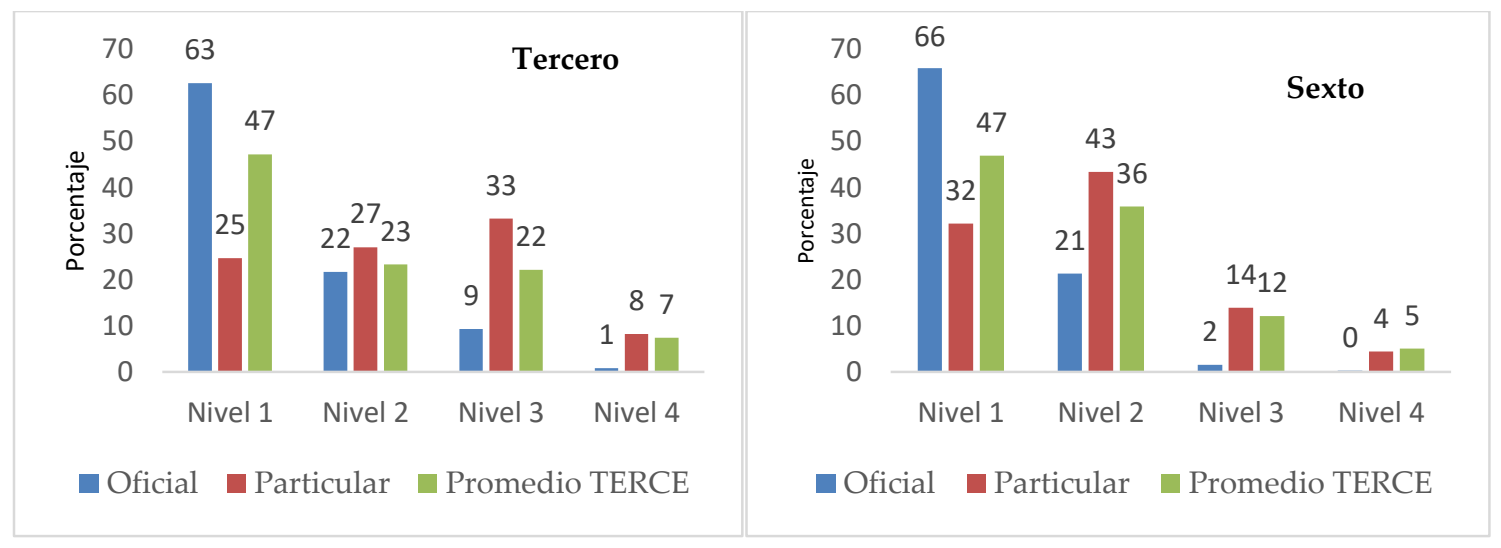

Figura 2. Distribución de los estudiantes por nivel de desempeño en la prueba de Matemática según grado, en Panamá. 
En la prueba de matemática de sexto grado (Figura 2), los estudiantes del sector oficial se distribuyen en un $66 \%$ en el nivel I. Esto significa que pueden ordenar números naturales y decimales, leen datos explícitos en tablas y gráficos, mientras que en los niveles II y III llega a $21 \%$ y $2 \%$, respectivamente (es decir, calculan área y perímetro, o resuelven problemas de proporcionalidad, por ejemplo). En el nivel IV no hay valor de porcentaje de estudiantes.

En contraste, en el sector particular, en el nivel I se observa un $25 \%$, en el nivel II un $27 \%$, mientras que en el nivel III hay un 33\% (que es nuevamente un valor superior al porcentaje promedio de todos los países participantes, de $23 \%$ ). En el nivel IV se observa un $8 \%$, valor ligeramente superior al promedio alcanzado por todos los países $(7 \%)$.

En la prueba de ciencias naturales de sexto grado (Figura 3), los estudiantes del sector oficial se distribuyen en un 54\% en el nivel I. Esto significa, por ejemplo, que reconocen acciones para satisfacer necesidades vitales o cuidados de la salud en contextos cotidianos. En el nivel II, un 35\% interpreta información simple en tablas y gráficos; el nivel III alcanza 10\%, es decir, el estudiante llega a conclusiones o las reconoce en trabajos de investigación; y, en el nivel IV sólo un 1\%, utiliza términos científicos para comprender procesos naturales, por ejemplo.

Ocurre de manera similar en el sector particular: en el nivel I se observa un valor de $18 \%$, en el nivel II de $41 \%$, en el nivel III hay un $27 \%$ (superior al porcentaje promedio de todos los países participantes, de 15\%) y en el nivel IV se observa un 15\% (también superior al promedio de todos los países, que fue de $6 \%$ ).

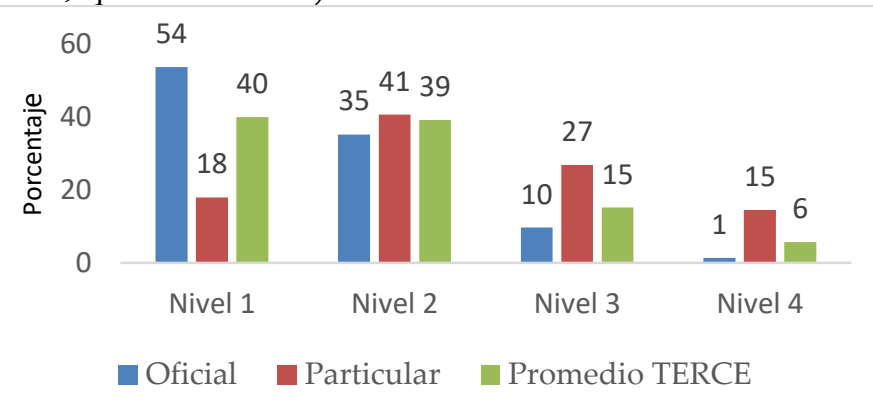

Figura 3. Distribución de estudiantes de sexto grado por nivel de desempeño en la prueba de ciencias naturales, Panamá.

\section{Factores asociados}

\section{Composición socioeconómica de los estudiantes}

En la Figura 4 se observa que el $75 \%$ de los estudiantes del sector oficial de tercero y sexto grado tiene un índice de nivel socioeconómico menor o igual a cero y el $50 \%$ de los estudiantes del sector particular de tercero y sexto grado tienen un índice de nivel socioeconómico superior a uno. 


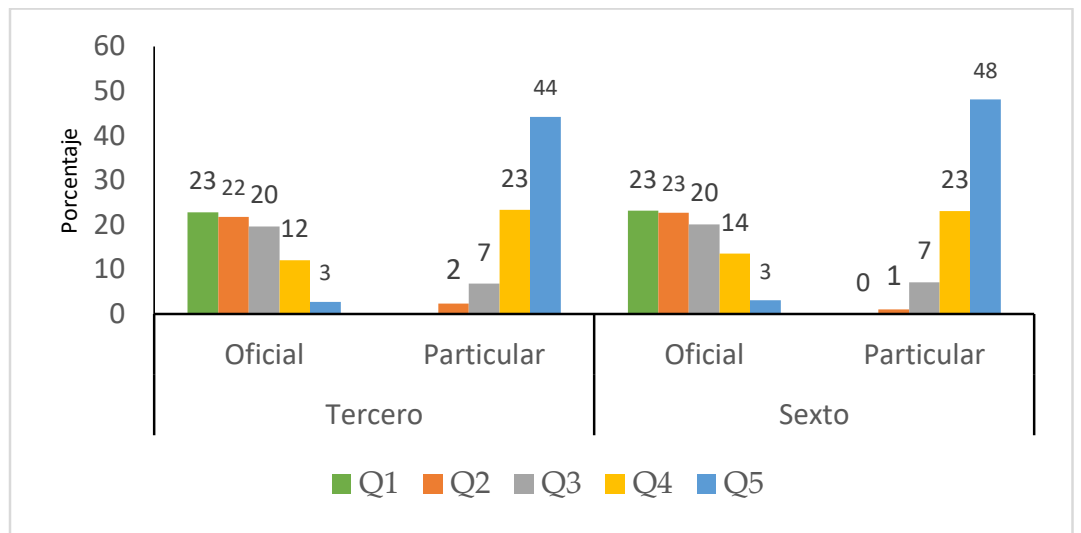

Figura 4. Porcentaje de estudiantes por sector y grado según quintil del índice de nivel socioeconómico, en Panamá.

Adicionalmente, el 65\% de los estudiantes de escuelas oficiales de tercero y sexto grado pertenece a los tres quintiles más bajos del índice de nivel socioeconómico, mientras que alrededor de un 70\% de estudiantes del sector particular están en los quintiles superiores del índice de nivel socioeconómico.

\section{Relación entre resultados y nivel socioeconómicos}

En la Figura 5 se observa que los puntajes promedios para cada quintil del índice de nivel socioeconómico de las pruebas de lectura y matemática de tercer grado en Panamá son inferiores para los estudiantes del sector oficial con respecto a los del sector particular. Además, el puntaje promedio de los estudiantes oficiales aumenta a medida que aumenta el índice de nivel socioeconómico.

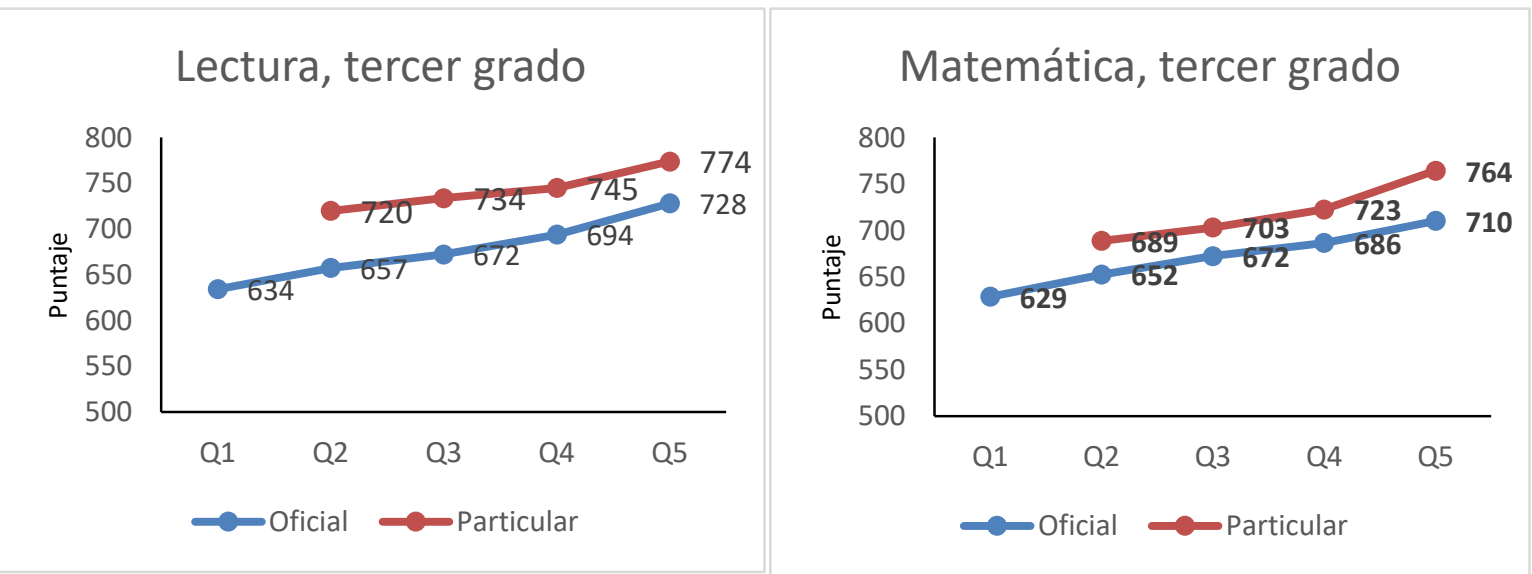

Figura 5. Puntajes promedio en Lectura y Matemática de estudiantes de tercer grado, según sector y quintil del índice de nivel socioeconómico, en Panamá. 
En la Figura 6 se muestra los puntajes promedios para cada quintil del índice de nivel socioeconómico de las pruebas de lectura, matemática y ciencias de sexto grado son inferiores para los estudiantes del sector oficial, con respecto a los del sector particular. Se evidencia cómo el puntaje promedio de los estudiantes de escuelas oficiales se incrementa a medida que aumenta el índice de nivel socioeconómico.

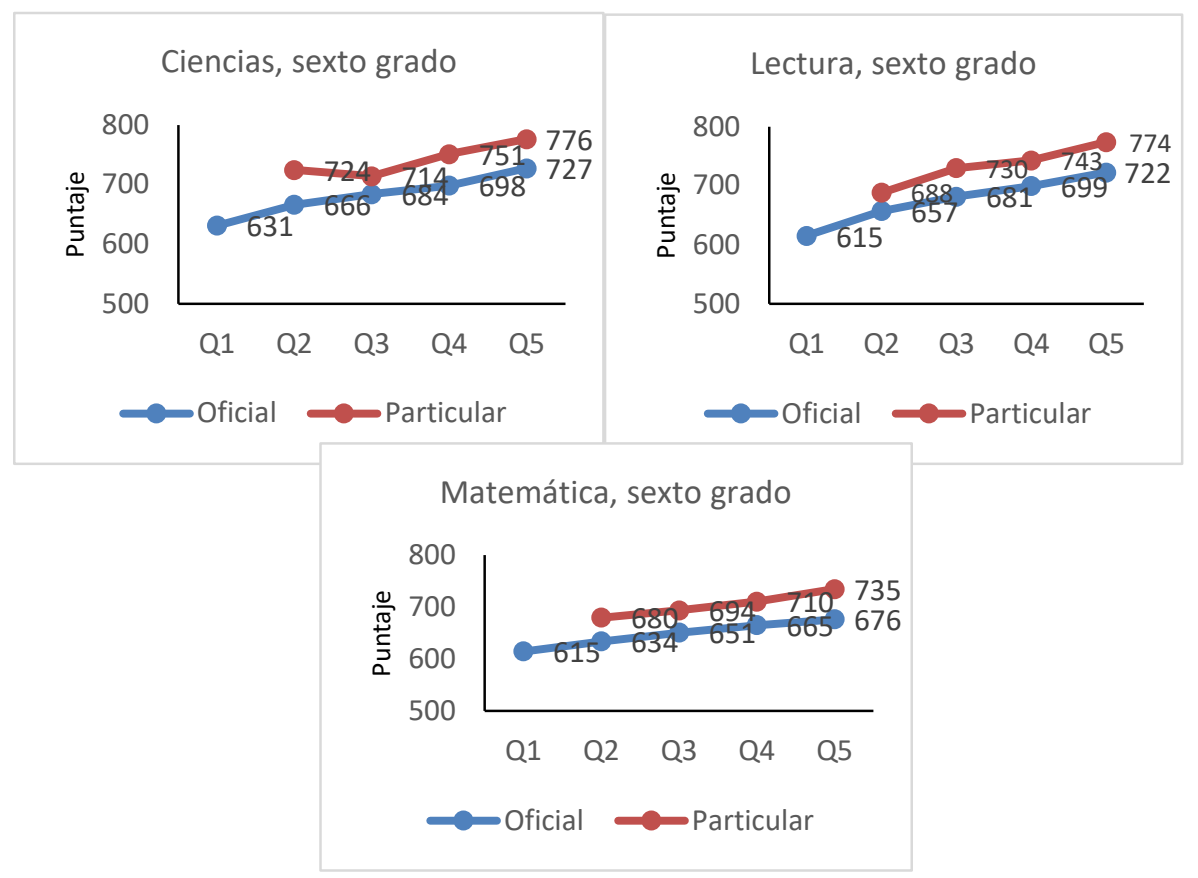

Figura 6. Puntajes promedio en Ciencias, Lectura y Matemática de estudiantes de sexto grado, según sector y quintil del índice socioeconómico, en Panamá.

Relación entre resultados y nivel socioeconómicos

\section{Aportes de la institución educativa al aprendizaje de los estudiantes}

La Tabla 2 muestra los ICC para cada prueba en los grados de tercero y sexto en el sector oficial. Estos valores indican que en tercer grado la prueba de matemática presenta mayor variación que la prueba de lectura. Esto implica que los resultados en matemática están muy posiblemente más influenciados por las características de las escuelas. Por otra parte, en sexto grado la prueba que presenta mayor variación es lectura.

De igual manera, se observa en las pruebas de lectura y matemática que la influencia de las características de las escuelas disminuye al aumentar el grado escolar. Esto significa que mientras mayor es el grado escolar, menos importante es la escuela para explicar las variaciones de rendimiento en las pruebas evaluadas. 
Tabla 2.

Correlación intra-clase (ICC) para modelos nulos por grado y prueba, en Panamá.

\begin{tabular}{l|l|l}
\hline \multirow{2}{*}{ Prueba del TERCE en Panamá } & Grado escolar \\
\cline { 2 - 3 } & Tercero & Sexto \\
\hline Lectura & 21.26 & 21.25 \\
Matemática & 27.71 & 17.63 \\
Ciencias & $*$ & 19.33 \\
\hline
\end{tabular}

*: No se aplicó prueba de ciencias en tercer grado en el TERCE Panamá.

En la prueba de lectura de tercero y sexto grado en las escuelas oficiales, el 21\% de la variación en los puntajes se explica por la acción de las escuelas. Además, en las pruebas de matemática la variación de los puntajes se explica por la acción de las escuelas en un $28 \%$ en tercer grado, y en un 17\% en sexto grado. En la prueba de ciencias de sexto grado, la variación de los puntajes se explica por las características de la escuela en un 19\%.

\section{Características de los actores escolares de escuelas oficiales y el desempeño en las pruebas}

En esta sección del estudio se analiza la asociación lineal entre factores a nivel de estudiante, del docente o del aula, así como a nivel de la escuela mediante diferentes coeficientes de correlación (Pearson, Eta y Gamma) para las escuelas oficiales.

\section{Tabla 3.}

Factores según actor escolar y su relación lineal con el promedio en las pruebas de lectura, matemática y ciencias, en tercero y sexto grado, en Panamá.

\begin{tabular}{ll}
\hline Nivel de estudiante & \\
\hline Factores & Relación \\
\hline Libros de textos & $+\mathrm{S}$ \\
Cuaderno & $+\mathrm{S}$ \\
Estudia y trabaja & $\mathrm{S}$ \\
Repitencia & $\mathrm{S}$ \\
Asistencia de los profesores & $\mathrm{S}$ \\
Sienten que los profesores están contentos & $+\mathrm{NS}$ \\
Motivados por sus profesores a seguir estudiando & $+\mathrm{S}$ \\
Los profesores les piden que expliquen sus respuestas & $+\mathrm{NS}$ \\
\hline Nivel docente & \\
\hline Nivel de estudio de los profesores & No relación lineal \\
Institución donde obtuvo el título de profesor & - NS \\
Cursos de perfeccionamiento & + NS
\end{tabular}


Observación del desempeño diario de los alumnos $\quad+$ NS

Grado de acuerdo con la motivación _ - NS

Nivel educativo más alto que alcanzará la mayoría de los alumnos - NS

Los estudiantes muestran respeto por sus compañeros + NS

Los directivos promueven que los docentes planifiquen sus clases + NS

\begin{tabular}{ll}
\hline Director & \\
\hline Años de trabajar como director & $+\mathrm{NS}$ \\
El docente tiene título de profesor & $+\mathrm{NS}$ \\
La escuela cuenta con sala de cómputo & $+\mathrm{NS}$ \\
La biblioteca tiene diferentes títulos & $+\mathrm{NS}$ \\
Se entrega libros de textos gratis & $+\mathrm{NS}$ \\
La escuela participa en programas focalizados de gestión escolar & $+\mathrm{NS}$ \\
La escuela participa en programas de competencias docentes & $+\mathrm{NS}$ \\
\hline
\end{tabular}

\section{Códigos:}

+ : relación lineal positiva.

-: $\quad$ relación lineal negativa.

S: $\quad$ relación estadísticamente significativa.

NS: no estadísticamente significativo/a.

En la Tabla 3, a nivel de estudiante, es posible observar que la asociación entre tener libro de texto de la asignatura y el puntaje en las pruebas es positiva y significativa. Los estudiantes que tienen libros de textos tienen mayor puntaje que los que no tienen. De igual manera, la asociación entre tener cuaderno para tomar notas y el puntaje en las pruebas es positiva y significativa; esto es, los estudiantes que tienen cuadernos para tomar notas tienen mayor puntaje que aquellos que no tienen.

La asociación entre estar motivado por sus docentes para seguir estudiando y el puntaje en las pruebas es positiva y significativa. Esto significa que los estudiantes que respondieron que nunca han sido motivados a seguir estudiando tienen menor puntaje que los que a veces son motivados. Por otro lado, la asociación entre "además de estudiar, trabajar" y el puntaje en las pruebas es negativa y significativa; esto implica que los estudiantes que además de estudiar trabajan tienen menor puntaje que los que no trabajan, como era de esperarse por la observación en campo. Otro factor que influye de manera negativa es la repitencia; esto significa que la asociación entre haber repetido un curso y el puntaje en las pruebas es negativa y significativa, es decir, los estudiantes que han repetido algún curso tienen menor puntaje que los que no han repetido.

A nivel de director y docente las asociaciones entre los diversos factores y los puntajes de pruebas no son estadísticamente significativas.

\section{Discusión}

El análisis presentado demuestra que los puntajes de las pruebas TERCE para Panamá fueron significativamente menores al promedio regional latinoamericano del TERCE. Al analizar los puntajes según sector, los puntajes de las pruebas para los estudiantes del sector particular fueron significativamente mayores al promedio regional TERCE, mientras que los puntajes de los estudiantes del sector oficial fueron significativamente menores que el promedio regional TERCE. Esto pone en 
clara evidencia las diferencias entre la educación del sector oficial y particular, con base estadística.

En la prueba de lectura, en el nivel I se concentró un 26\% de estudiantes en tercero y un 18\% en sexto grado; es decir, cerca de un $8 \%$ más que el promedio TERCE. En el nivel II, son valores de $21 \%$ y $52 \%$, en tercero y sexto, respectivamente. Esto implica que los estudiantes sólo identifican información explícita de un texto, sin lograr una reflexión o la emisión de juicios sobre lo leído. Esto llevó al equipo investigador a indicar que una de las tareas prioritarias del maestro en el aula es formar estudiantes como "lectores competentes". También refleja la importancia de la formación inicial y continua de los maestros, además de dotar de herramientas metodológicas que logren llevar a los estudiantes a niveles más altos hacia una lectura realmente comprensiva.

En matemática, en el nivel de desempeño I, se espera que los alumnos lean datos explícitos arreglados en tablas y gráficas; allí se concentra un $63 \%$ en tercer grado y un $66 \%$ en sexto grado. En los niveles III y IV, en donde se espera que el estudiante resuelva problemas más complejos que involucren, por ejemplo, números naturales, comparación y conversión de medidas, o bien interpretación de elementos de figuras geométricas, se observa tan solo un 10\% de estudiantes de escuelas oficiales de tercer grado y un $3 \%$ de estudiantes de sexto grado.

Finalmente, en la prueba de ciencias naturales, alrededor de 54\% y 35\% están en los niveles I y II, respectivamente. Esto, por su parte, implica que los estudiantes solo son capaces de reconocer, en el nivel I, los cuidados de la salud y necesidades vitales en contextos cotidianos, mientras que en el nivel II son sólo capaces de interpretar información básica, como, por ejemplo, la presentación de estructuras en tablas y gráficos, la clasificación de seres vivos, o bien, su descripción y presentación de relaciones de causa y efectos en situaciones muy familiares. El 11\% de los estudiantes de sexto grado en escuelas oficiales logró alcanzar los niveles III y IV: esto significa que son capaces de interpretar información variada en diferentes formatos (gráficos, tablas, entre otros), aplican sus conocimientos científicos para explicar fenómenos de la naturaleza, y discriminan de entre distintas preguntas, aquellas que se pueden responder científicamente, entre otras habilidades.

Todo lo anteriormente expuesto, lleva al equipo investigador a pensar y cuestionar (hacia fines constructivos) el efecto e impacto de las capacitaciones masivas que realiza el Ministerio de Educación, cada año. Más aún, un estudio de casos realizado por Jackson, Estrella y Rodríguez (2017) indica que el maestro es muy "tradicional": al observar las aulas se evidenció que en sexto grado, en la clase de matemática y ciencias, un $13.3 \%$ y y $5.9 \%$ del tiempo, respectivamente, el alumno sólo copia (ya sea del tablero a su cuaderno, o de un libro al cuaderno), lo cual coloca al estudiante en una posición pasiva. Tampoco se evidenció con alta frecuencia la lectura en voz alta en las clases en español. En el mismo estudio de casos, se observó que en matemática, español y ciencias alrededor de un 46.7\%, $30.0 \%$ y $23.5 \%$ del tiempo, respectivamente, el estudiante desarrolla ejercicios cortos de manera individual, sin evidenciarse el debate o la reflexión o la argumentación de los resultados de estas actividades (2017).

Otro aspecto importante a resaltar es que los datos arrojan que menor es la influencia que ejerce la escuela en el aprendizaje cuando el grado aumenta; esta instancia es más evidente en el área de matemática. Esto debe llevar a una reflexión no sólo sobre la práctica docente, sino también sobre cómo la escuela enfrenta el determinismo social. Sobre esto hay un artículo relevante escrito por Tedesco (2016), en que se afirma que, en las evaluaciones, aparte de dar diferencias estadísticas poco 
significativas, el resultado más fuerte es que el determinismo social afecta el rendimiento de los aprendizajes y que la escuela tiene poca capacidad de romper dicho determinismo. Es por ello necesario realizar estudios que contemplen además los aspectos de equidad social y educativa. El trabajo a futuro del equipo investigador irá en esta dirección y línea de investigación.

A nivel de alumno, se observa que los recursos que se utilizan en el aula (libros y cuadernos) son variables que inciden de manera positiva y directa en los resultados; esto permite deducir que los estudiantes de bajos recursos, que en la mayoría de los casos sólo tienen un cuaderno, tendrían en la generalidad de los casos, pocas posibilidades de lograr buenos resultados. En complemento a este aspecto identificado, el estudio de casos en escuelas oficiales de Panamá (arriba mencionado, Jackson et al., 2017) mostró que los recursos que utiliza generalmente el maestro son el tablero, el cuaderno para el estudiante y las copias de libros de textos. Esto se identificó transversalmente en todas las aulas observadas (2017, pp. 61-63). De aquí deriva una recomendación de que el Ministerio de Educación debe continuar e intensificar la dotación a las escuelas en contextos más desfavorables de recursos para el aula.

Por otro lado, la repitencia es una variable que tiene influencia negativa sobre los logros de aprendizaje; esta variable debe permitir analizar con profundidad las alternativas que el Ministerio de Educación facilita, como por ejemplo: los programas de recuperación académica ${ }^{3}$, los módulos que los estudiantes desarrollan durante el trimestre, y las pruebas de recuperación que se aplican a los estudiantes de noveno y duodécimo grado. En términos generales, estas alternativas nacionales son provechosas, pero no están surtiendo el efecto deseado, que es el aprendizaje efectivo.

En cuanto al docente, su nivel de estudios y la institución donde éste obtuvo el título son variables que no son significativas en el logro de aprendizaje. Esto implica que es necesario reflexionar y realizar estudios más profundos en lo que se refiere a la formación de los maestros, revisar el pénsum académico y el perfil de ingreso a las carreras docentes, ya que la formación inicial del maestro es vital en la profesión docente. Basado en estas reflexiones, el equipo de investigadores continuará su línea de investigación y, en general, su aporte a la sociedad panameña.

\section{Agradecimiento}

Los autores dejan constancia de su agradecimiento a la SENACYT (Secretaría Nacional de Ciencia, Tecnología e Innovación), por el cofinanciamiento recibido para la conducción del estudio en Panamá. El cofinanciamiento proviene del proyecto adjudicado y financiado por la Dirección de Aprendizaje de la Ciencia, APR SENACYT. Se agradece al Ministerio de Educación de Panamá (MEDUCA), por su oportuna colaboración; a los docentes y estudiantes que apoyaron el estudio en las escuelas; y a la Universidad del Caribe.

\footnotetext{
${ }^{3}$ El Programa de Recuperación Académica en Panamá, va dirigido a aquellos estudiantes que fracasan en una, dos o
} tres asignaturas; se imparte durante el verano del año calendario en Panamá. 


\section{Referencias}

Agencia de la Calidad de la Educación (2015). Las escuelas que hacen la diferencia: evidencia de la prueba TERCE 2013 en la construcción de comunidades enfocadas en el aprendizaje. Santiago de Chile.

División de Evaluación de Logros de Aprendizajes. (2012). Metodología de Construcción de Grupos Socioeconómicos. Agencia de la Calidad de Educación. Recuperado de http://www.agenciaeducacion.cl/wp- content/uploads/2013/02/Metodologia-deConstruccion-de-Grupos-Socioeconomicos-SIMCE-2011.pdf.

Jackson, D., Estrella, J. y Rodríguez, W. (2017). Enseñando en contextos desfavorables. Estudio de casos de cuatro escuelas oficiales de Panamá. Panamá.

Flotts, M. P., Manzi, J., Jiménez, D., Abarzúa, A., Cayuman, C., \& García, M. J. (2015). Informe de resultados TERCE: logros de aprendizaje. UNESCO Publishing.

Murillo, F. y Hernández-Castilla, R. (2011). Efectos escolares de factores socio-afectivos: un estudio multinivel para Iberoamérica. Revista de Investigación Educativa (RIE), 29-2, 407-427. Recuperado de: https://www.proceso.com.mx/523357/el-inee-sin-metodo-para-evaluar-aprendizaje-de-alumnosindigenas-consejera-schmelkes

Sancho, C., González Such, J. y Bakieva, M. (2014). PSPP. Correlación bivariada. Coeficiente de Pearson. Innovamide L4U. Red de Innovación Educativa y Calidad Docente. Elaboración y evaluación de materiales de aprendizaje. Universitat de València, España. Recuperado de: http://www.uv.es/innovamide/14u/PSPP/pspp09/pspp09.wiki

StataCorp (2013). Stata Statistical Software: Release 13. College Station, TX: StataCorp LP.

Tedesco, J. C. (2016). Diez notas sobre los sistemas de evaluación de los aprendizajes.

Torrecilla, M. y Carrasco, R. (2011). ¿La escuela o la cuna? Evidencias sobre su aportación al rendimiento de los estudiantes de América Latina. Estudio multinivel sobre la estimación de los efectos escolares. Profesorado. Revista de Currículo y Formación de Profesorado, 15(3): 27-50.

Treviño, E. (2006). Evaluación del aprendizaje de los estudiantes indígenas en América Latina: desafíos de medición e interpretación en contextos de diversidad culturaly desigualdad social. Revista Mexicana de Investigación Educativa, ISSN 1405-6666. 11(28): 225-268.

Warren, W.G. (1971). Correlation or Regression: Bias or Precision. Appl Stat. 1971;20(2):148. 